SHORT COMMUNICATION



\title{
Challenges and distribution of radiographic diagnosis in dogs at a tertiary veterinary institution
}

\author{
A Olatunji-Akioye $^{1 *}$, o Omotosho ${ }^{2} \&$ F Bolaji-Alabi $^{1}$ \\ 1. Department of Veterinary Surgery and Radiology, Faculty of Veterinary Medicine, University of Ibadan, Nigeria \\ 2. Department of Veterinary Medicine Faculty of Veterinary Medicine University of Ibadan, Nigeria
}

*Correspondence: Tel.: +2348034091407; E-mail: bonik2001@yahoo.com

\begin{abstract}
Copyright: (C) 2018 Olatunji-Akioye et al. This is an open-access article published under the terms of the Creative Commons Attribution License which permits unrestricted use, distribution, and reproduction in any medium, provided the original author and source are credited.
\end{abstract}

Publication History: Received: 19-06-2018 Accepted: $30-10-2018$

\begin{abstract}
Radiography is an important clinical diagnostic tool in any Veterinary establishment and especially for a tertiary institution. It is however limited by lack of functional equipment and staff. This study attempts to assess the challenges faced by the Radiology Unit of the Surgery Clinic and enumerate the distribution of cases at a tertiary veterinary centre between August 2016 and June 2017. All the referred cases to the unit within the study period were accessed $(n=52) .26$ were selected based on the inclusion criteria which were a radiography request from a clinician and an actual return to clinic of a clear radiograph. Age, sex, lesions and clinical history were recorded from case files and radiographs. The age of animals presented ranged from one month to 13 years. Eleven (11) representing $42.3 \%$ of the dogs were female while $14(53.8 \%)$ were male, and one animal's sex was omitted. Eighteen (18) were single lesions while four presented with multiple lesions, and four had no observable lesions. Fourteen 14 (53.8\%) were fracture cases of long bones either for diagnosis or postreduction evaluation. Three (11.5\%) were to check for suspected thoracic metastasis in cases where lumps were found in other parts of the body. Four (15.3\%) were of the pelvis to diagnose hip dysplasia. Seven $(26.9 \%)$ were to aid diagnosis in cases in which swellings were palpated within the abdomen and one (3.8\%) was of the cervical spine while another was of a foot (3.8\%). The implication of lack of radiographic equipment and staff problems in a tertiary veterinary centre were elucidated and ideal conditions suggested.
\end{abstract}

Keywords: Diagnosis, Dogs, Radiography, Surgery, Tertiary veterinary centre, University

\section{Introduction}

Radiography is an imaging modality that utilizes $x$ rays to produce images of internal organs based on the degree of attenuation and contrast (Chen \& Whitlow, 2010). It is particularly useful for bones and areas where there is varying contrast and the opacity of the organs is used in evaluating what is assessed. In order of decreasing opacity, there is bone, soft tissue, fat, fluid and gas opacity. Plain radiography relies on natural and physical contrast based on the density of material through which the x-ray radiation must pass (Chen \& Whitlow, 2010). Radiography may be used plain or for contrast studies where substances are used to increase or decrease the contrast of a part leading to improved visualization (Andreucci et al., 2014). Clinical diagnosis with radiography gives definitive diagnosis and improves the accuracy of surgical intervention in cases such as fractures. 
Retrospective and prospective studies are essential to determine prevalence of diseases in a particular geographic location (Chaves et al., 2014). It also helps clinicians to improve the diagnosis and treatment of common ailments in any Veterinary Teaching Hospital (an example of a tertiary veterinary institution) (Libardoni et al., 2016). Ideally, a tertiary Veterinary establishment should be equipped with facilities that will make it function effectively in the diagnosis, treatment and care of animal diseases. While all efforts are being made to ensure that this is done, there are challenges which make this impossible. A glaring example is lack of a functional diagnostic radiology unit.

This study attempts to chronicle the challenges that may impede radiographic diagnosis in dogs by assessing the distribution of radiographic cases over a ten month period spanning August 2016 and June 2017 using a tertiary veterinary institution in Nigeria as a case study.

\section{Materials and Methods}

Cases that were referred to the Surgery Unit of a tertiary veterinary centre were reviewed. The single inclusion criterion was if a clear radiograph was returned to the clinic after a radiograph request was given by a clinician of a clear radiographic image. Twenty-six (26) cases met the inclusion criteria of all the fifty-two cases presented during the study period. The radiographs were peer-viewed and classified based on sex, age, number of lesions, type of lesion, part affected, and whether radiograph was diagnostic or not within the period being assessed. The frequency of the types of requests and radiographic diagnoses were calculated and statistical analysis of each was performed by imputing the data into Microsoft Excel, (2010

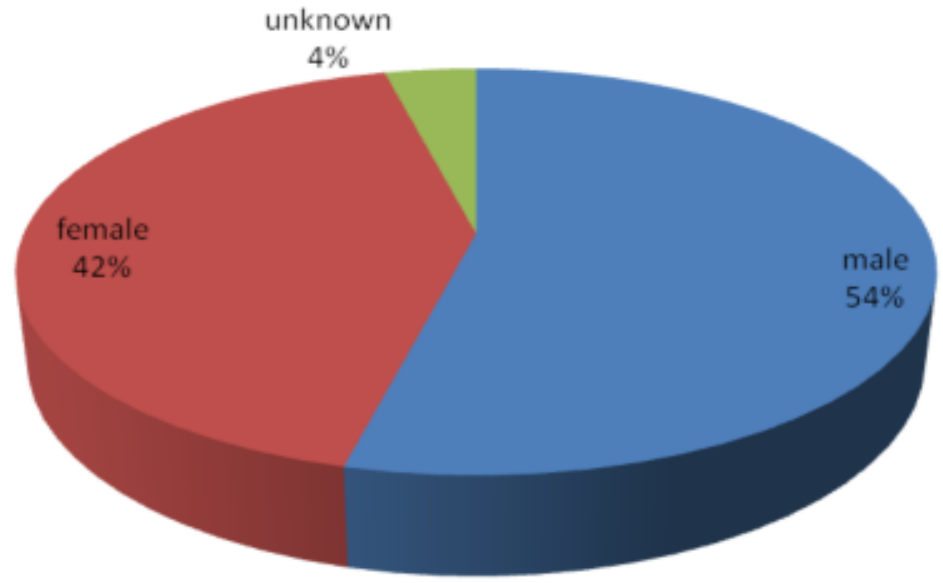

Figure 1: Sex distribution of radiographic diagnosis in dogs at a tertiary veterinary centre between August 2016 and June 2017 version) and means calculated. The machinery available at the tertiary veterinary centre were evaluated and the challenges faced by clients and clinicians were reviewed and enumerated.

\section{Results and Discussion}

Out of the twenty-six cases included in this study based on the inclusion criteria, the clinical records showed most of the animals (69\%) were young but the age ranged from one month to thirteen years. Results also showed 11 (42.3\%) of the dogs were female while 14 (53.8\%) were male, and one (1) animal's sex was not recorded (Figure 1). Eighteen dogs were diagnosed to have single lesions and four had multiple lesions bringing the number of lesions to thirty, the remaining four (4) had no observable lesions either because they were absent or because the radiographs were not diagnostic (Figure 2). Four (4) representing $13 \%$ of the lesions were to evaluate treatment while twenty-six (26) representing $87 \%$ were to facilitate diagnosis. Fourteen (14) representing $47 \%$ were to diagnose fractures while three (3) representing $10 \%$ were to check for metastasis to the thorax due to swellings found in other parts of the body. Four (4) representing 13\% were to diagnose hip dysplasia; seven (7) representing $23 \%$ were to diagnose abdominal swellings while one (1) representing $3 \%$ was to diagnose cervical spine anomaly and one (1) representing $3 \%$ was to diagnose the foot (Figure 3 ). Of the fourteen (14) fractures, nine (9) representing $64 \%$ were in femur while three (3) representing $21 \%$ were in radius and ulna bone and two (2) representing $14 \%$ were in tibia and fibula bone.

All radiographs were acquired from human radiography centres outside the immediate environment of the tertiary veterinary centre which serve as referral centres to the Surgery Unit of the Veterinary Teaching Hospital. The machinery at the hospital which consisted of one (1) unit stationary machine, two (2) units table top machines and one (1) unit mobile $x$-ray machine were all inaccessible. The staff challenges at the human centres were collated via oral interviews and included poor images as a result of improper positioning and a subsequent predisposition to retakes of the radiographs and multiple exposure of animals to primary x-ray beams. Staff 
lack of knowledge of animal handling and positioning techniques, fear of the animals and inability to manage the animals lead to requests that owners restrain their animals and a resultant exposure to the potential hazard of the primary $x$-ray beams, as seen in some of the reviewed radiographs (Plate I).

The diagnostic challenges being faced at this tertiary veterinary institution is significant because these institutions are supposed to serve as a last line of medical diagnosis for primary and secondary veterinary centres. The uniqueness of radiographic diagnosis which has no substitute is also important. While diagnosis of soft tissue lesions may be done by some other imaging modality, fractures can hardly be diagnosed by other methods. The distribution of cases presented at the Radiology Unit shows that lesions like fractures especially of the long bones were more frequently encountered than in other bone types and soft tissues. This finding is in agreement with Simon et al. (2010) who found that femoral fractures were more common due to the fact that the distal half of the femur is away from the long axis of the pelvic limb making it more susceptible to bending forces leading to fractures. Libardoni et al. (2016), in a larger sample size retrospectively studied, over a 10-year period reported that tibia and fibula fractures were next in incidence to femoral fractures. In our 10-month study, we observed a similar pattern of higher incidence of femoral fractures but next in incidence were radio-ulnar fractures. This further supports earlier reports of a higher tendency of fractures in long bones. This establishes the fact that cases require radiographic diagnosis and the need to make available on-site, machinery that will make the safe

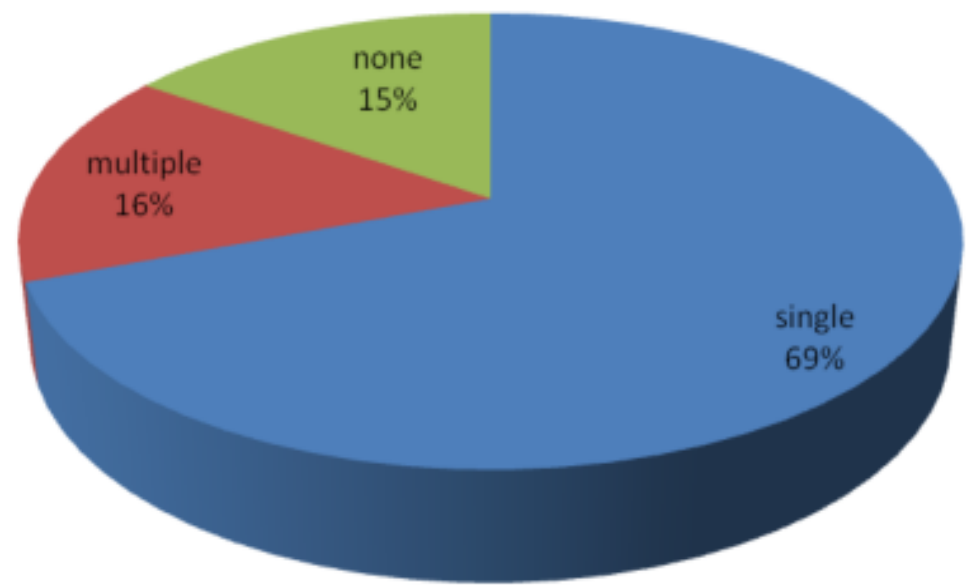

Figure 2: Radiographic diagnosis of lesions in dogs at a tertiary veterinary centre between August 2016 and June 2017

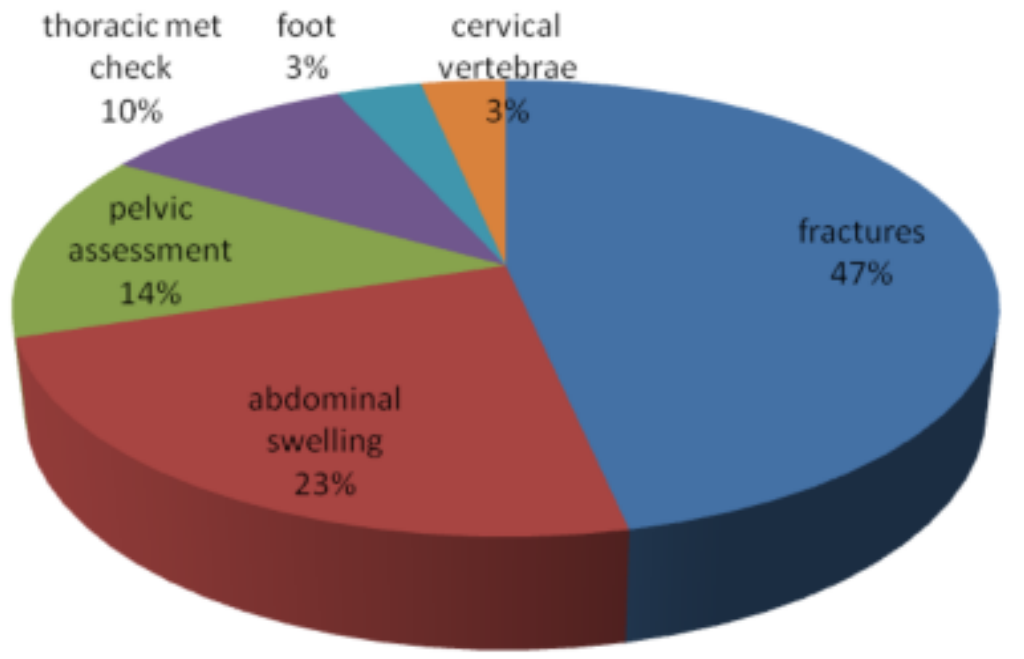

Figure 3: Lesion distribution through radiography in dogs at a tertiary veterinary centre, between August 2016 and June 2017

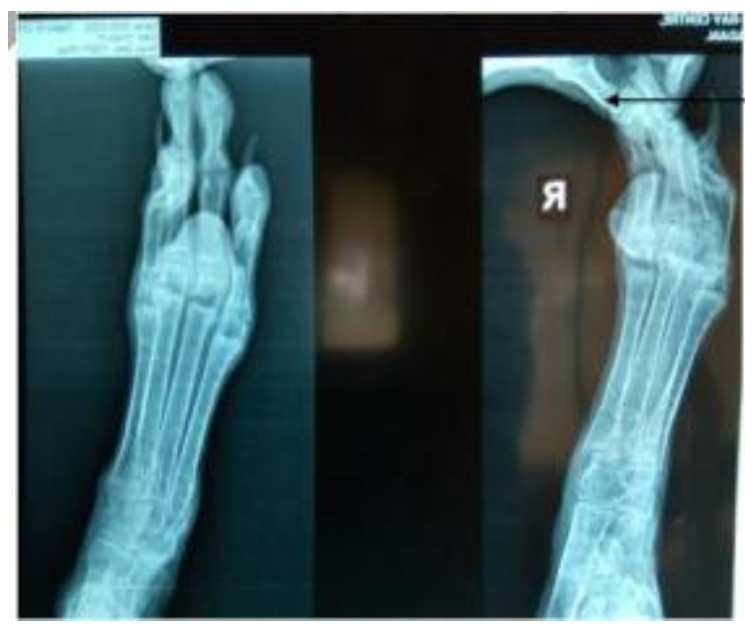

Human hand for restraint

Plate I: Radiation safety issues with dog radiographs from human centres 
acquisition of radiographs possible in tertiary veterinary institutions like the Veterinary Teaching Hospital.

Of all the radiographs assessed, none was acquired at the tertiary veterinary centre, these radiographs had been acquired at human centres around the campus. A tertiary Veterinary institution should have a stationary machine, for use in small animals and a mobile machine that will be useful for large animal and ambulatory cases. A trained veterinary radiographer and animal handlers to assist in animal restraint are also a necessity.

Radiation safety is paramount as excessive active or passive exposure is known to increase the risk of longer term effects such as cancers (Wall et al., 2006). In this study, it was observed that several of the radiographs showed human body parts which constitute a radiation safety challenge. The owners/guides of the dogs are made to restrain them during the procedure as the facilities and personnel at the human radiograph centres are not trained to handle animal patients (Plate I). To forestall future exposure of clients to this health hazard, and minimize the retakes of radiographs due to poor radiographs, there is an urgent need to fund the VTH and provide the radiographic equipment and Veterinary Radiology expertise needed to perform the top class care it was established to provide. The present adopted practice of taking radiography requests out of the VTH complex to a human centre may contribute to the high rate (50\%) of non-return of radiographs by clients issued radiographic request forms.

While several factors like economics and cost may account for some of the cases not returning to the clinic, with improved on-site facilities, more cases will be available to teach students which forms part of the mandate of a tertiary Veterinary care centre, Veterinary Teaching Hospital and Veterinary establishments.

\section{Acknowledgement}

We would like to acknowledge all colleagues in the Departments of Veterinary Medicine and Surgery and Radiology who provide clinical services to the Veterinary Teaching Hospital and use Radiography in the course of diagnosis of their cases as this has helped to improve interest in and use of Radiography.

\section{References}

Andreucci M, Solomon R \& Tasanarong A (2014). Side effects of radiographic contrast media: Pathogenesis, risk factors, and prevention. BioMed Research International 741018. doi.org/10.1155/2014/741018

Chen MYM \& Whitlow CT (2010). Scope of Diagnostic Imaging. In Basic Radiology, second edition, McGraw -Hill Prefessional. Pgs 1-15

Chaves OR, Beckmann DV, dos Santos RP, Aiello G, Andrades AO, Baumhardt R, Silveira LB \& Mazzanti A, (2014). Neurological diseases in dogs examined at the Veterinary Teaching Hospital of the Federal University of Santa Maria, RS: 1.184 cases (2006-2013). Pesquisa Veterinária Brasileira, 34(10): 9961001.

Libardoni RN, Serafini GMC, Oliveira C, Schimites PI, Chaves OR, Feranti JPS, Costa CA, Amaral AS, Raiser AS \& Soares AV (2016). Appendicular fractures of traumatic etiology in dogs: 955 cases (2004-2013) Ciencia Rural, 46(3): 542-546.

Simon S, Ganesh MR, Ayyapan S, Rao GD, Kumar R, Kundave VR \& Das BC (2010). Incidences of pelvic limb fractures in dogs: A survey of 478 cases. Veterinary World 3(3): 120-121.

Wall BF, Kendall GM, Edwards AA, Bouffler $S$, Muirhead CR \& Meara JF, (2006). Review Article: What are the risks from medical Xrays and other low dose radiation? The British Journal of Radiology, 79, 79(940):285-294. 